\title{
Direct and Inverse Scattering for Transient Electromagnetic Waves in Nonlinear Media
}

\author{
Gerhard Kristensson \\ Department of Electromagnetic Theory \\ Lund Institute of Technology, P.O. Box 118 \\ S-221 00 Lund, Sweden \\ David J.N. Wall \\ Department of Mathematics and Statistics \\ University of Canterbury, Christchurch \\ New Zealand
}

No. 153

June, 1997

Keywords. Nonlinear wave propagation, inverse problem, wave splitting, Kerr effect in fibre optics. 


\begin{abstract}
Nonlinear propagation of electromagnetic waves is an important problem in optics. Often the properties of the nonlinear media are not fully understood. The solution of an inverse problem can provide an aid to that understanding.

An inverse transmission problem is posed, it is one of reconstructing the medium parameters, by measurement of a wave that has been propagated through the nonlinear medium. The nonlinear medium is assumed to be homogeneous and isotropic. The methods have application to nonlinear optics, and the numerical results for both the direct and inverse problems presented are based on the nonlinear Kerr effect, which is observed in the optical wavelength band. However, the mathematical techniques that are developed are applicable to any set of nonlinear first order equations. The method is therefore model independent.
\end{abstract}

\title{
1 Introduction
}

The effects of dissipation, in a passive nonlinear optical media, impose constraints on the form that the constitutive relations can take. This is utilised to examine the allowable form of the constitutive equations in $\S 2$.

One of the major results, given in this paper, is to show that a system of first order partial differential equations can be effectively analysed via a single one-way wave equation. This is a significant result as it enables exact solutions to be formulated for both the direct and the inverse problems.

In $\S 2$, we state the basic equations governing one-dimensional transmission through a nonlinear medium. It is shown, in $\S 3$, that by wave splitting the Maxwell equations describing one-dimensional wave propagation through an isotropic, dissipative nonlinear optical medium can be reduced to two one-way wave (transport) equations. It is further shown that for the problem of relevance to this paper, that consideration of just one oneway wave equation is necessary. This is because the wave splitting only allows coupling of the two one-way wave equations through the wavespeed functional.

The method of characteristics is then employed to solve both the direct transmission problem and the inverse transmission problem i.e., the problem of finding the functional form of the wavespeed function after having measured the transmitted field. The various nonlinear models for optical media that are used in $\S 5$ are presented in $\S 4$. Our techniques in $\S 5$ build on those discussed in (Connolly and Wall 1997) and we will henceforth refer to this paper as (I). Further problems involving source reconstruction for a linear one-way wave equation, are considered in (Wall and Lundstedt 1996). In $\S 5$, we pose an inverse transmission problem of reconstructing the medium parameters from measurement of a propagated wave through the nonlinear medium.

One of the novel features of our inverse problems is that the inverse solution can be written down in explicit form. Another novel feature is that the solution also depends continuously on the given transmission data, and this is proven in $\S 5$. Prior to discovering this inverse problem, we were of the opinion that perhaps all non-trivial inverse problems are ill-posed; this solution has modified our opinion.

Our methods have application to nonlinear optics and the numerical results for both the direct and inverse problems presented in $\S 5.1$ are based on the nonlinear Kerr effect

\footnotetext{
${ }^{0} 1995$ Physics and Astronomy Classification Scheme PACS: 02.30Jr, 03.50De, 42.50Rh, 42.81Dp

${ }^{0}$ Keywords: Nonlinear wave propagation, inverse problems, wave splitting, Kerr effect.

${ }^{0}$ July 1997
} 
which is observed in the optical wavelength band. The analysis and results presented in this paper are valid upto, but not beyond, any wavefunction shock or discontinuity that may occur because of utilisation of the Kerr model. When a more acurate model such as the Raman model is utilised dispersion prevents formulation of the shock; the various available models are discussed further in $\S 2$.

\section{Basic equations}

We first review here some of the nonlinear effects as observed in fibre optics. When the electromagnetic wave propagates through a dielectric medium then polarization of the medium molecules occurs. This polarization is generally represented through the polarization field, $\boldsymbol{P}$, which is related to the electric flux density, $\boldsymbol{D}$, and the electric field intensity, $\boldsymbol{E}$, as

$$
\boldsymbol{D}=\epsilon_{0} \boldsymbol{E}+\boldsymbol{P}
$$

where $\epsilon_{0}$ is the electric permittivity of free space. In general, evaluation of $\boldsymbol{P}$ will require a quantum-mechanical approach. Although such an approach is often necessary, when the optical frequencies are near the resonance of the medium, a classical approach can be used far from medium resonance. This will be the case for optical fibres in the wavelength range $0.5-2 \mu \mathrm{m}$, and this is the range that is of interest in the study of nonlinear effects in optical fibres. The polarization vector can be split into a linear and a nonlinear part as

$$
\boldsymbol{P}=\boldsymbol{P}^{l}+\boldsymbol{P}^{n l}
$$

with the linear part being represented by a susceptibility kernel, $\chi^{(1)}$, through

$$
P_{i}^{l}(\boldsymbol{x}, t)=\epsilon_{0} \int_{-\infty}^{t} \chi_{i j}^{(1)}\left(t-t^{\prime}\right) E_{j}\left(\boldsymbol{x}, t^{\prime}\right) d t^{\prime}
$$

and the nonlinear part as (see e.g., (Agrawal 1995; Shen 1995), and (Shubert and Wilhelmi 1986))

$$
\begin{gathered}
P_{i}^{n l}(\boldsymbol{x}, t)=\epsilon_{0} \int_{-\infty}^{t} \int_{-\infty}^{t} \int_{-\infty}^{t} \chi_{i j k l}^{(3)}\left(t-t_{1}, t-t_{2}, t-t_{3}\right) E_{j}\left(\boldsymbol{x}, t_{1}\right) E_{k}\left(\boldsymbol{x}, t_{2}\right) \\
\times E_{l}\left(\boldsymbol{x}, t_{3}\right) d t_{3} d t_{2} d t_{1}
\end{gathered}
$$

In these equations, the fields are assumed to be quiescent before a fixed time, and are dependent on the spatial coordinates, $\boldsymbol{x}=(x, y, z) \in \mathbb{R}^{3}$, and the temporal variable, $t \in \mathbb{R}$, and where Cartesian tensor notation has been used to represent the tensor fields; so that $i, j, k, l \in\{1,2,3\}$. Note that causality causes the upper limit of the integrals, in the previous equations, to be truncated. Also observe that in general, polarisation effects produce memory effects. The lowest order nonlinear response, which is shown here, allows for the molecular vibrations, which is attributed to the Raman effect. In general, both the electrons and the nuclei respond to the optical field, but the nuclei respond slower than the electrons. Often the nonlinear effect is modelled by assuming an instantaneous response as

$$
P_{i}^{n l}(\boldsymbol{x}, t)=\epsilon_{0} \chi_{i j k l}^{(3)} E_{j}(\boldsymbol{x}, t) E_{k}(\boldsymbol{x}, t) E_{l}(\boldsymbol{x}, t)
$$


and this amounts to neglecting the Raman effect. For silica fibres the vibrational or Raman response occurs over a time scale of $60-70 \mathrm{fs}$; this will mean that the equation (2.1) will be approximately valid for pulse widths greater than 1ps. This is the model of the Kerr effect utilised in this paper in $\S 4.1,4.2$, and 5.1.

Non-resonant intensity dependent effects (incoherent) can be included by use of a nonlinear susceptibility kernel of the form

$$
\chi_{i j k l}^{(3)}\left(t-t_{1}, t-t_{2}, t-t_{3}\right)=\chi_{i k l}^{(3)} R_{j}\left(t-t_{1}\right) \delta\left(t-t_{2}\right) \delta\left(t-t_{3}\right)
$$

with

$$
\int_{0}^{\infty} R_{j}(t) d t=1
$$

and then

$$
P_{i}^{n l}(\boldsymbol{x}, t)=\epsilon_{0} \chi_{i k l}^{(3)} E_{k}(\boldsymbol{x}, t) E_{l}(\boldsymbol{x}, t) \int_{-\infty}^{t} R_{j}\left(t-t^{\prime}\right) E_{j}\left(\boldsymbol{x}, t^{\prime}\right) d t^{\prime}
$$

The response function $R_{j}$ should include electronic and vibrational (Raman) contributions, and if the electronic effect is assumed to be instantaneous then (for notational convenience we omit the index $j$ )

$$
R(t)=\left(1-f_{r}\right) \delta(t)+f_{R} h_{R}(t)
$$

with

$$
h_{R}=\frac{\tau_{1}^{2}+\tau_{2}^{2}}{\tau_{1} \tau_{2}^{2}} \exp \left(-t / \tau_{2}\right) \sin \left(t / \tau_{1}\right)
$$

where the $R$ function parameters, $f_{r}, f_{R}, \tau_{1}$, and $\tau_{2}$ are chosen so that the main memory effect is for $t<0.4 \mathrm{ps}$ (for silica fibres). The form of this model, $h_{R}$, is the well known Lorentz or resonance model. Extensions of our methods in $\S 5$ for this model will be considered in a later paper.

The Maxwell equations, in a source-free region, linking the electric field, $\boldsymbol{E}$, magnetic field intensity, $\boldsymbol{H}$, with the magnetic and electric flux densities, $\boldsymbol{B}$, and $\boldsymbol{D}$, respectively are

$$
\left\{\begin{array}{l}
\nabla \times \boldsymbol{E}(\boldsymbol{x}, t)=-\frac{\partial}{\partial t} \boldsymbol{B}(\boldsymbol{x}, t) \\
\nabla \times \boldsymbol{H}(\boldsymbol{x}, t)=\frac{\partial}{\partial t} \boldsymbol{D}(\boldsymbol{x}, t)
\end{array}\right.
$$

Furthermore, assume that all fields depend only on the coordinate $z$. The Maxwell equations then become

$$
\left\{\begin{array}{l}
\mathrm{J} \cdot \frac{\partial}{\partial z} \boldsymbol{E}(z, t)=-\frac{\partial}{\partial t} \boldsymbol{B}(z, t) \\
\mathrm{J} \cdot \frac{\partial}{\partial z} \boldsymbol{H}(z, t)=\frac{\partial}{\partial t} \boldsymbol{D}(z, t)
\end{array}\right.
$$


where $\mathbf{J}=\hat{z} \times \mathbf{I}$ (a rotation $\pi / 2$ around the axis $\hat{z}$ ), where $\mathbf{I}$ is the identity operator. Assuming only transverse components of the electric field and non-magnetic materials, rewrite these equations as ${ }^{1}$

$$
\left\{\begin{array}{l}
\frac{\partial}{\partial z} \boldsymbol{E}(z, t)=\frac{1}{c_{0}} \eta_{0} \frac{\partial}{\partial t} \mathrm{~J} \cdot \boldsymbol{H}(z, t) \\
\eta_{0} \frac{\partial}{\partial z} \mathrm{~J} \cdot \boldsymbol{H}(z, t)=\frac{1}{c_{0} \epsilon_{0}} \frac{\partial}{\partial t} \boldsymbol{D}(z, t)
\end{array}\right.
$$

where the parameter $\eta_{0}=\sqrt{\mu_{0} / \epsilon_{0}}$ is the impedance of free space, and $c_{0}=1 / \sqrt{\mu_{0} \epsilon_{0}}$ is the wavespeed of electromagnetic radiation in free space.

The Maxwell equations imply the Poynting theorem

$$
\nabla \cdot S+E \cdot \partial_{t} D+H \cdot \partial_{t} B=0
$$

where the Poynting vector $S$ is defined as

$$
\boldsymbol{S}=\boldsymbol{E} \times \boldsymbol{H}
$$

Let the constitutive relations specifying the relationship between the field intensities and the field flux densities be written as

$$
\left\{\begin{array}{l}
\boldsymbol{D}(\boldsymbol{x}, t)=\epsilon_{0} \mathcal{F}(\boldsymbol{E}(\boldsymbol{x}, \cdot))(t) \\
\boldsymbol{B}(\boldsymbol{x}, t)=\mu_{0} \boldsymbol{H}(\boldsymbol{x}, t)
\end{array}\right.
$$

and be assumed to hold in each point in space. We assume for optical media the polarization effect is purely due to the electric field and can be represented through an operator $\mathcal{F}$. Then, the first of these equations is a generalization of those considered earlier. Dissipation of the medium implies, through integration of the Poynting theorem over space and time that (Karlsson and Kristensson 1989)

$$
\mathcal{E}(t) \equiv \int_{-\infty}^{t}\left\{\boldsymbol{E}\left(t^{\prime}\right) \cdot \partial_{t} \boldsymbol{D}\left(t^{\prime}\right)+\boldsymbol{H}\left(t^{\prime}\right) \cdot \partial_{t} \boldsymbol{B}\left(t^{\prime}\right)\right\} d t^{\prime} \geq 0, \quad \text { for all fields }
$$

Notice that this dissipation constraint includes only the electromagnetic part of dissipation. If conversion of electromagnetic energy into mechanical energy, or other energy forms, takes place in the medium, the dissipation constraints will look differently.

In the remainder of the paper we assume that the medium is isotropic. This implies that $\boldsymbol{D}$ and $\boldsymbol{E}$ have the same field orientation, therefore scalar quantities suffice. It follows from (2.4) that the appropriate functional inequality to study is

$$
\int_{-\infty}^{t} E\left(t^{\prime}\right) \frac{d}{d t} \mathcal{F}(E)\left(t^{\prime}\right) d t^{\prime} \geq 0, \quad \text { for all } E \in \mathcal{X}
$$

where the operator $\mathcal{F}: \mathcal{X} \rightarrow \mathcal{X}$, and where the affine function space $\mathcal{X}$ is defined by

$$
\mathcal{X}=\left\{f \in C^{1}(\mathbb{R}): f(t)=0, t<t_{0}, t_{0} \text { fixed }\right\}
$$

\footnotetext{
${ }^{1}$ Note that $\mathbf{J} \cdot \mathbf{J}=-\mathbf{I}_{2}$, where $\mathbf{I}_{2}$ is the identity operator in the $x$-y-plane.

${ }^{2}$ As then, $D_{i}=\epsilon_{0} \mathcal{F}\left(E_{i}\right)$.
} 
for $t_{0}$ bounded. It is further assumed that a Banach algebra is defined on $\mathcal{X}$, then integration by parts gives

$$
E(t) \mathcal{F}(E)(t)-\int_{-\infty}^{t} E^{\prime}\left(t^{\prime}\right) \mathcal{F}(E)\left(t^{\prime}\right) d t^{\prime} \geq 0
$$

where the prime, on the dependent variable, has been used to denote differentiation with respect to its independent variable, $t$. We can convert this expression into a more convenient operator inequality by defining an operator $\mathcal{G}: \mathcal{X} \rightarrow \mathcal{X}$ as

$$
\mathcal{G}(E)(t)=\int_{-\infty}^{t} E^{\prime}\left(t^{\prime}\right) \mathcal{F}(E)\left(t^{\prime}\right) d t^{\prime}
$$

Then

$$
\frac{d}{d t} \mathcal{G}(E)(t)=E^{\prime}(t) \mathcal{F}(E)(t)
$$

and the energy equality can then be rewritten as

$$
\frac{E}{E^{\prime}} \frac{d}{d t} \mathcal{G}(E)-\mathcal{G}(E) \geq 0, \quad \text { for all } E \in \mathcal{X}
$$

We observe that if the electric field is independent of $t$, then the inequality reduces to a vacuous equality.

\subsection{Constitutive relations of the function type - finite dimensional case}

The operator $\mathcal{F}$ is acting on the function $E$. However, as this case will not be considered further in this paper, we just consider the finite dimensional case here.

Let $F$ be a real, continuous function ${ }^{4}$ defined on $\mathbb{R}$, i.e., $F \in C(\mathbb{R})$. In this case $\mathcal{F}(E)(t)=F(E(t))$ is just a functional composition. So replacing the notation, $\mathcal{F}$ by $F$, and $\mathcal{G}$ by $G$, the basic inequality, (2.6), becomes

$$
E(t) G^{\prime}(E(t))-G(E(t)) \geq 0, \quad \text { for all } E \in \mathcal{X}
$$

where the prime on $G$ denotes differentiation with respect to its argument, and where $G \in C^{1}(\mathbb{R})$ is a primitive function to $F$, i.e., $G^{\prime}(E)=F(E)$, such that $G(0)=0$. Therefore, in this finite-dimensional case, the relevant question to ask to ensure that the media is dissipative is:

Question: For what functions $G(x)$ is the following inequality satisfied:

$$
x^{2} \frac{d}{d x}\left(\frac{G(x)}{x}\right)=x G^{\prime}(x)-G(x) \geq 0, \quad x \in \mathbb{R}
$$

This inequality is equivalent to

$$
\frac{d}{d x}\left(\frac{G(x)}{x}\right) \geq 0, \quad x \in \mathbb{R}
$$

\footnotetext{
${ }^{3}$ We denote by $C^{n}(\Omega)$ the space of $n$ times continuously differentiable functions on $\Omega$.

${ }^{4}$ Note that $F \in C(\mathbb{R})$ is sufficient for Proposition 2.1, but stronger conditions will be required in the remainder of the paper, c.f., Definition 2.1.
} 
Proposition 2.1 If $F$ is an increasing function, with $F \in C(\mathbb{R})$, for all $x \in \mathbb{R}$, then $F$ defines a constitutive relation that satisfies the energy inequality (2.7).

Proof: Since $F(x)$ is a increasing function, we have $F(x) \leq F(y)$, whenever $x \leq y$. From this we easily get

$$
\begin{gathered}
\int_{0}^{x} F(t) d t \leq \int_{0}^{x} F(x) d t=x F(x), \quad x \geq 0 \\
-\int_{0}^{x} F(t) d t \geq \int_{x}^{0} F(x) d t=-x F(x), \quad x \leq 0
\end{gathered}
$$

or

$$
\int_{0}^{x} F(t) d t \leq x F(x), \quad \text { for all } x \in \mathbb{R}
$$

Moreover, this inequality is equivalent to $(2.7)$; in fact, with $G(x)=\int_{0}^{x} F(t) d t$ we have $x G^{\prime}(x) \geq G(x)$.

The converse to the Proposition 2.1, that every function that satisfies the energy inequality (2.7) is an increasing function, is not true. A simple counter example is $F(x)=$ $.8 x+\sin x$.

It is readily seen that if $F \in C^{1}(\mathbb{R})$, the conditions of Proposition 2.1 are satisfied if $F^{\prime}>0$, i.e., $F$ is a strictly increasing function. In the sequel, we need a slightly stronger condition which motivates the following definition:

Definition $2.1 A$ dissipative medium is said to be positively dissipative iff $F \in C^{1}(\mathbb{R})$ and $F^{\prime}(x) \geq a>0$, i.e., it is a strictly increasing function with a derivative bounded away from zero.

Media that are positively dissipative have the important property that $F^{\prime}(x) \geq a>0$, i.e., $F^{\prime}$ is bounded below, this will be important in $\S 3$. In particular, in this paper we will only consider problems for media that are positively dissipative.

We now consider some models for nonlinear media which are dissipative.

Example 1 - Kerr polynomial model: Let $G(x)=\sum_{n=1}^{N} a_{n} x^{n}$, with $N$ bounded, then the inequality $(2.7)$ reduces to

$$
\sum_{n=1}^{N}(n-1) a_{n} x^{n-2} \geq 0, \quad x \in \mathbb{R}
$$

This will be satisfied if the coefficients $a_{n}$ of the polynomial satisfy (sufficient conditions)

$$
\left\{\begin{array}{l}
a_{1}, \quad \text { arbitrary } \\
a_{n} \geq 0, \quad n \text { even } \\
a_{n}=0, \quad n \text { odd }
\end{array}\right.
$$

It follows that $F(x)=G^{\prime}(x)=\sum_{n=1}^{N} n a_{n} x^{n-1}$. This is a generalisation of the polynomial relation that is the commonly used for the constitutive model of the Kerr effect, c.f., equation (2.1) and see (Landau, Lifshitz, and Pitaevskii 1984), i.e.,

$$
D_{i}=\epsilon_{0}\left(\epsilon_{1} E_{i}+\frac{1}{3} \epsilon_{3} E_{i}^{3}\right) \quad i \in\{1,2,3\}
$$

\footnotetext{
${ }^{5}$ See Footnote 4 on page 5.
} 
implies

$$
F(x)=\epsilon_{1} x+\frac{1}{3} \epsilon_{3} x^{3}
$$

The result proven here shows this model is guaranteed positively dissipative if $\epsilon_{1}$ and $\epsilon_{3}$ are positive constants, c.f., $\S 4.1$.

Example 2 - Kerr saturation model: This model, generalises the Kerr effect to include the possibility that for high values of electric field, the nonlinearity saturates. A rational polynomial model for this can be written as

$$
F(x)=\epsilon_{1} x+\frac{\alpha x^{3}}{x^{2}+\beta^{2}}
$$

which implies

$$
F^{\prime}(x)=\epsilon_{1}+\frac{\alpha x^{4}+3 \alpha \beta^{2} x^{2}}{\left(x^{2}+\beta^{2}\right)^{2}}
$$

It then follows that this model is guaranteed positively dissipative if $\epsilon_{1}$ and $\alpha$ are positive constants, c.f., also $\S 4.2$.

\section{$3 \quad$ Wave splitting}

Throughout the sequel, we assume that the medium is homogeneous and isotropic and then the constitutive relations become (2.3)

$$
\left\{\begin{array}{l}
D_{i}(\boldsymbol{x}, t)=\epsilon_{0} \mathcal{F}\left(E_{i}(\boldsymbol{x}, \cdot)\right)(t) \\
B_{i}(\boldsymbol{x}, t)=\mu_{0} H_{i}(\boldsymbol{x}, t)
\end{array} \quad i \in\{1,2,3\}\right.
$$

The assumption on the electromagnetic field components in $\S 2$ implies that the electric and magnetic vector components lie in a plane tranverse to the $z$-axis (or the $x_{3}$-axis). Let $w_{1}$ be the transverse component of the electric field $\boldsymbol{E}$, and $w_{2}$ the corresponding component of the field $\eta_{0} \mathrm{~J} \cdot \boldsymbol{H}$, then we can utilise these equations, and (3.1), in (2.2), to yield the partial differential equation system

$$
\frac{1}{c_{0}} \frac{\partial}{\partial t}\left(\begin{array}{c}
\mathcal{F}\left(w_{1}\right)(z, t) \\
w_{2}(z, t)
\end{array}\right)=\left(\begin{array}{ll}
0 & 1 \\
1 & 0
\end{array}\right) \frac{\partial}{\partial z}\left(\begin{array}{c}
w_{1}(z, t) \\
w_{2}(z, t)
\end{array}\right)
$$

When the constitutive relations are of the function case of $\S 2.1$, then this system of equations can be expressed as

$$
\frac{1}{c_{0}}\left(\begin{array}{c}
F^{\prime}\left(w_{1}\right) \partial_{t} w_{1} \\
\partial_{t} w_{2}
\end{array}\right)=\left(\begin{array}{ll}
0 & 1 \\
1 & 0
\end{array}\right) \frac{\partial}{\partial z}\left(\begin{array}{l}
w_{1} \\
w_{2}
\end{array}\right)
$$

For positively dissipative media, Definition 2.1 ensures that $F \in C^{1}(\mathbb{R}), F^{\prime}\left(w_{1}\right) \geq a>0$, so we can rewrite this equation as

$$
\frac{1}{c_{0}}\left(\begin{array}{c}
\sqrt{F^{\prime}\left(w_{1}\right)} \partial_{t} w_{1} \\
\partial_{t} w_{2}
\end{array}\right)=\left(\begin{array}{cc}
0 & \frac{1}{\sqrt{F^{\prime}\left(w_{1}\right)}} \\
\frac{1}{\sqrt{F^{\prime}\left(w_{1}\right)}} & 0
\end{array}\right)\left(\begin{array}{c}
\sqrt{F^{\prime}\left(w_{1}\right)} \partial_{z} w_{1} \\
\partial_{z} w_{2}
\end{array}\right)
$$


This form suggests that a transformation of the dependent variable will enable this system to be expressed in a more convenient form. To facilitate this, introduce the anti-derivative of $\sqrt{F^{\prime}\left(w_{1}\right)}$, through

$$
g^{\prime}(x)=\sqrt{F^{\prime}(x)}
$$

Let it also be assumed that $g$ be subject to $g(0)=0$, so that

$$
g(x)=\int_{0}^{x} \sqrt{F^{\prime}(t)} d t
$$

The properties just assumed for $g^{\prime}$ (through Definition 2.1), and the inverse function theorem, ensures the $C^{1}$ function $g$ has an inverse $g^{-1}$.

Proposition 3.1 The function $g$ is a homeomorphism, if it is associated with an F function from a constitutive relation, for a positively dissipative medium.

Proof: For $F$ a positively dissipative function, $F \in C^{1}(\mathbb{R}), F^{\prime}(x) \geq a>0$, then from elementary analysis - with $F$ strictly increasing, this will imply $g^{\prime}$ is strictly increasing and hence the existence, and continuity of $g^{-1}$, which is also strictly increasing. In fact we have more, $g$ is a diffeomorphism, but this is not required later.

Now by defining new dependent variables $u \in \mathbb{R}^{2}$

$$
u=\left(\begin{array}{l}
u_{1} \\
u_{2}
\end{array}\right)=\left(\begin{array}{c}
g\left(w_{1}\right) \\
w_{2}
\end{array}\right)
$$

the underlying partial differential equation can be transformed from (3.2) into

$$
u_{t}=A(u) u_{z}
$$

The symmetric matrix $A(u)$ is

$$
A(\boldsymbol{u})=c_{0}\left(\begin{array}{cc}
0 & \frac{1}{g^{\prime}\left(g^{-1}\left(u_{1}\right)\right)} \\
\frac{1}{g^{\prime}\left(g^{-1}\left(u_{1}\right)\right)} & 0
\end{array}\right) \equiv c\left(u_{1}\right)\left(\begin{array}{ll}
0 & 1 \\
1 & 0
\end{array}\right)
$$

where the wavespeed $c\left(u_{1}\right)$ is

$$
c\left(u_{1}\right)=\frac{c_{0}}{g^{\prime}\left(g^{-1}\left(u_{1}\right)\right)}=c_{0} \frac{d}{d u_{1}} g^{-1}\left(u_{1}\right)
$$

The following result is necessary for applications in the remainder of this paper.

Proposition 3.2 With $g$, associated with an $F$ in the positively dissipative class, the wavespeed $c \in C(\mathbb{R})$ is a strictly decreasing function of field amplitude.

Proof: Observe $F \in C^{1}$ is a strictly increasing function. So from Proposition 3.1, and as $c(\xi)=c_{0} / g^{\prime}\left(g^{-1}(\xi)\right)$, it follows $c$ is strictly decreasing.

The positively dissipative medium assumption, ensures that the function $g^{\prime}(x)$ is a positive function bounded away from zero, i.e., $F^{\prime}(x) \geq a>0$, for all $x \in \mathbb{R}$. Furthermore, this implies that $c$ is a globally bounded above function. The matrix $A$ is therefore bounded in the standard operator norm, specifically, sup $\|A(u)\| \leq c_{0} / \sqrt{a}$. We collect this result for later reference in the following proposition: 
Proposition 3.3 With $g$, associated with an $F$ in the positively dissipative class, the matrix-valued operator is bounded, as $\sup \|A(\boldsymbol{u})\| \leq c_{0} / \sqrt{a}$.

The system (3.4) is symmetric hyperbolic, so when the function $c$ and its first derivative are globably bounded, the Cauchy problem for the equation (3.4) has at most one classical short time solution. This is shown in (Kreiss and Lorenz 1989, Lemma 5.1.1) for the case where the solution has periodic initial conditions, and we extend their result to apply for our problem in Appendix A. However it should be noted that not all media have first derivatives of $c$ that are globally bounded, although two of the models we consider in $\S 4.1$ and $\S 4.2$ do possess this property. In Theorem 3.1 we prove existence and uniqueness of the one-way wave equation, which is shown to be central to our development later in this section, for a wider range of media.

Equation (3.4) can now, in a special case, be solved by the method of characteristics, after it has been converted to a diagonal form. This diagonalisation can be motivated physically by the concept of wave splitting; this is because under certain simplifying hypotheses the dependent variables can be then interpreted as waves propagating in two opposite directions. To this end, introduce two new dependent variables $u^{ \pm}(z, t)$ defined by

$$
\left(\begin{array}{l}
u^{+} \\
u^{-}
\end{array}\right)=\frac{1}{2}\left(\begin{array}{cc}
1 & -1 \\
1 & 1
\end{array}\right)\left(\begin{array}{l}
u_{1} \\
u_{2}
\end{array}\right)=P\left(\begin{array}{l}
u_{1} \\
u_{2}
\end{array}\right)
$$

where the transformation matrix has an inverse such that,

$$
\left(\begin{array}{l}
u_{1} \\
u_{2}
\end{array}\right)=\left(\begin{array}{cc}
1 & 1 \\
-1 & 1
\end{array}\right)\left(\begin{array}{l}
u^{+} \\
u^{-}
\end{array}\right)=P^{-1}\left(\begin{array}{l}
u^{+} \\
u^{-}
\end{array}\right)
$$

Substitution of these new dependent variables into (3.4) yields dynamics for the new fields $u^{ \pm}(z, t)$ as

$$
\begin{aligned}
\partial_{t}\left(\begin{array}{l}
u^{+} \\
u^{-}
\end{array}\right) & =P A\left(u^{+}, u^{-}\right) P^{-1} \partial_{z}\left(\begin{array}{l}
u^{+} \\
u^{-}
\end{array}\right) \\
& =c\left(u^{+}+u^{-}\right)\left(\begin{array}{cc}
-1 & 0 \\
0 & 1
\end{array}\right) \partial_{z}\left(\begin{array}{l}
u^{+} \\
u^{-}
\end{array}\right)
\end{aligned}
$$

It should be observed that the two component waves still couple, through the functional wavespeed $c\left(u^{+}+u^{-}\right)$, despite the fact that the wavespeed matrix is diagonal. The effect of this coupling is to change the slope of the characteristic traces for each of the two wave equations. This has the useful interpretation that if at some time there is no reflected wave, there will continue to be no reflected wave for all future time, while the forward wave is modified by its own presence in the medium.

Of interest for the remainder of this paper is a wave propagation problem in a half space, $z \geq 0$. Appropriate initial and boundary values for this problem are; see Figure 1:

$$
\left.\begin{array}{l}
u^{+}(z, 0)=f(z) \\
u^{-}(z, 0)=0
\end{array}\right\} \quad z \geq 0, \quad u^{+}(0, t)=h(t), \quad t \geq 0
$$

Provided the partial differential equations specified in (3.6) satisfy the side conditions of equation (3.7), the system has a unique solution, see Theorem A.1. This implies that 


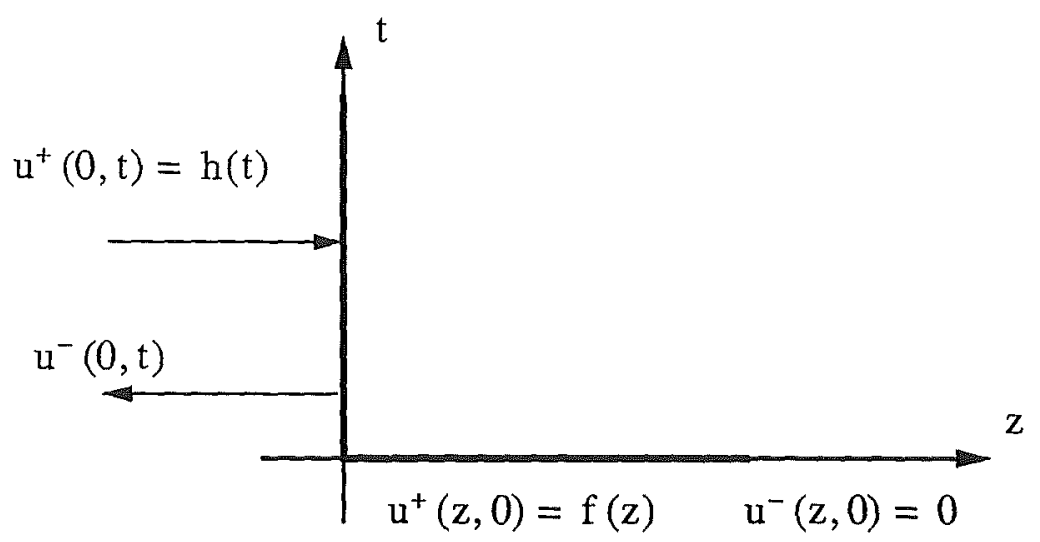

Figure 1: The geometry for the initial-boundary value problem.

$u^{-}(z, t) \equiv 0$, for all $z \geq 0$ and $t \geq 0$. Then the forward propagating wave field $u^{+}(z, t)$ satisfies the quasi-linear transport equation

$$
\partial_{t} u^{+}+c\left(u^{+}\right) \partial_{z} u^{+}=0
$$

which can be solved by the methods of characteristics. This means that the techniques utilised in (I) can be applied to the system of first order partial differential equations considered in this paper.

\subsection{Solution of the one-way wave equation}

We first examine when the one-way wave equation (3.8) has a classical solution. This enables us to find a solution to the direct propagation problem for the partial differential equation (3.4) with side conditions (3.7). For this section consider the initial-boundary value problem on the quarter plane $\left\{(z, t) \in \mathbb{R}^{2} \mid z>0, t>0\right\}$ for the one-way wave equation (3.8) with side conditions

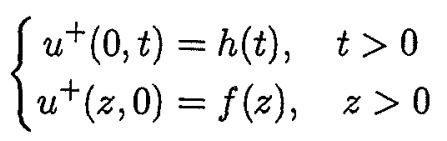

Sufficient conditions on the boundary value $h(t)$, and on the initial condition $f(z)$, for the classical solution of the direct propagation problem to exist, is found in (I); we weaken those conditions here. Let $\Gamma$ be a curve in the $t$ - $z$-plane, parameterized by the parameter $\zeta$, and defined by $t=\tau(\zeta)$ and the solution of

$$
\frac{d \tau(\zeta)}{d \zeta}=\frac{1}{c\left(u^{+}(\zeta, \tau(\zeta))\right)}
$$

Then, by (3.8), the variation in $u^{+}$along $\Gamma$ is

$$
\frac{d}{d \zeta} u^{+}(\zeta, \tau(\zeta))=D_{1} u^{+}(\zeta, \tau(\zeta))+\frac{1}{c\left(u^{+}(\zeta, \tau(\zeta))\right)} D_{2} u^{+}(\zeta, \tau(\zeta))=0
$$

where $D_{1} u^{+}$, and $D_{2} u^{+}$, denote the partial derivatives of $u^{+}$with respect to its first, and second arguments, respectively. This equation shows that $u^{+}$is constant along $\Gamma$, and that the characteristics defined by (3.9) are therefore straight lines. 
The characteristic trace (not necessarily unique) that goes through the point $(z, t)$, $t>0$ can be described by

$$
\tau(\zeta ; z, t)=t+\frac{\zeta-z}{c(h(\tau(0 ; z, t)))}
$$

Observe, for future reference, that the characteristic trace has slope 1/c. Later, we also require the inverse of $t^{\prime}=\tau(\zeta ; z, t)$, namely $\zeta=\tau^{-1}\left(t^{\prime} ; z, t\right)$ - the existence of which is assured for.c $>0-$ (see ( $\AA$ berg, Kristensson, and Wall 1996) for further use of this notation).

To simplify the notation in the remainder of this section, denote $t^{\prime}=\tau(0 ; z, t)$ by $\tau(z, t)$, where this is the intercept of the curve, $\Gamma$, which passes through the point $(z, t)$, with the line $z=0$. To determine $\tau=\tau(z, t)$ for a given value of $(z, t)$, it is necessary to solve the equation ${ }^{6}$

$$
(t-\tau) c(h(\tau))=z
$$

In the previous analysis, it is assumed that $\tau(z, t)>0$. Similar arguments can be used to find the solution when $\tau(z, t)<0$, i.e., when the characteristic trace is back propagated from $(z, t)$ and it cuts the $z$-axis prior to the $t$-axis. In this case, the pertinent transcendental equation is

$$
t c(f(\zeta))+\zeta=z
$$

where the solution $\zeta=\zeta(z, t)>0$ is the $z$-value where the characteristic curve intercepts the $z$-axis.

The solution of the direct propagation problem, $u^{+}$, is then determined from the boundary value or the initial condition as

$$
u^{+}(z, t)= \begin{cases}f(\zeta(z, t)) & \text { if } \tau(z, t)<0 \\ h(\tau(z, t)) & \text { if } \tau(z, t)>0\end{cases}
$$

The solution of the direct propagation problem, $u^{+}$, is then determined from the boundary value or the initial condition as

$$
u^{+}(z, t)= \begin{cases}f(\zeta(z, t) & \text { if } \tau(z, t)<0 \\ h(\tau(z, t)) & \text { if } \tau(z, t)>0\end{cases}
$$

An extension of this analysis we formulate as an uniqueness and existence theorem.

Theorem 3.1 Let $f, h \in C^{1}[0, \infty)$, and $c \in C^{1}(\mathbb{R})$ be given functions such that $f(0)=$ $h(0)$ and $c \geq c_{1}>0$, and let $T \geq 0$ and $Z \geq 0$ be determined by

$$
\begin{aligned}
& \frac{1}{Z}= \begin{cases}0 & \text { if } \frac{d}{d t} c(h(t)) \leq 0 \text { for all } t \geq 0 \\
\sup _{t \geq 0}\left\{-\frac{d}{d t} \frac{1}{c(h(t))}\right\} & \text { otherwise }\end{cases} \\
& \frac{1}{T}= \begin{cases}0 & \text { if } \frac{d}{d z} c(f(z)) \geq 0 \text { for all } z \geq 0 \\
\sup _{z \geq 0}\left\{-\frac{d}{d z} c(f(z))\right\} & \text { otherwise }\end{cases}
\end{aligned}
$$

\footnotetext{
${ }^{6}$ Obtained by setting $\zeta=0$ in equation (3.10).
} 
Furthermore, let $\tau(z, t)=\tau$ and $\zeta(z, t)=\zeta$, for a given value of $(z, t)$, be determined by

$$
\left\{\begin{array}{l}
(t-\tau) c(h(\tau))=z \\
t c(f(\zeta))+\zeta=z
\end{array}\right.
$$

and let

$$
\begin{cases}\Omega_{1}=\{(z, t): \tau(z, t)>0, & 0 \leq z \leq Z\} \\ \Omega_{2}=\{(z, t): \tau(z, t)<0, & 0 \leq t \leq T\}\end{cases}
$$

Then in the two regions $\Omega_{1}$ and $\Omega_{2}$, the continuously differentiable function, $u^{+}(z, t)$, defined by

$$
u(z, t)= \begin{cases}h(\tau(z, t)) & (z, t) \in \Omega_{1} \\ f(\zeta(z, t)) & (z, t) \in \Omega_{2}\end{cases}
$$

is the unique classical solution to

$$
\partial_{t} u+c(u) \partial_{z} u=0 \quad \text { subject to } \quad \begin{cases}u(z, 0)=f(z) & z \geq 0 \\ u(0, t)=h(t) & t \geq 0\end{cases}
$$

in the respective regions $\Omega_{1}$ and $\Omega_{2}$.

Proof : First examine the restricted region $\Omega_{1}$, and observe that the characteristic trace can be written as in equation (3.10). If two such traces intersect within the $(z, t)$ quarter plane and they originate from the $z=0, t>0$ line, with values $t_{1}$ and $t_{2}$, it is easily shown that they intersect at $Z$ where

$$
\frac{1}{Z}=-\frac{1}{t_{2}-t_{1}}\left(\frac{1}{c\left(h\left(t_{2}\right)\right)}-\frac{1}{c\left(h\left(t_{1}\right)\right)}\right)=-\left.\frac{d}{d t} \frac{1}{c(h(t))}\right|_{t=\tilde{t}} \quad \text { for some } \widetilde{t} \in\left[t_{1}, t_{2}\right]
$$

In such a case the region $\Omega_{1}$ is less than the whole quarter plane, and the largest value of the right hand side determines the smallest value of $z$ where a shock can occur, and the result follows; this occurs e.g., when $c^{\prime}<0$, and $h^{\prime}<0$. Consideration of the traces emanating from the $t=0$ line, and using a similar argument defines $\Omega_{2}$ and the time $T$.

To prove that (3.15) is a uniquely defined solution to our problem we need to use the implicit function theorem. For $(z, t) \in \Omega_{1}$, the first equation in (3.14) has a unique solution, by the implicit function theorem. In fact if $F_{1}(z, t ; \tau)=(t-\tau) c(h(\tau))-z=0$, then for $(z, t) \in \Omega_{1}$, which implies $z \leq Z$,

$$
\begin{aligned}
D_{3} F_{1}(z, t ; \tau)= & -c(h(\tau))+(t-\tau) \frac{d}{d \tau} c(h(\tau)) \\
& =-c(h(\tau))\left(z \frac{d}{d \tau} \frac{1}{c(h(\tau))}+1\right) \leq-c(h(\tau))\left(-\frac{z}{Z}+1\right)<0
\end{aligned}
$$

where $D_{3}$ denotes differentiation with respect to the third variable. This proves that for all $(z, t) \in \Omega_{1}$, there is a unique solution $\tau=\tau(z, t)$ to (3.14). Similarly, $F_{2}(z, t ; \zeta)=$ $t c(f(\zeta))+\zeta-z=0$, gives for $(z, t) \in \Omega_{2}$

$$
D_{3} F_{2}(z, t ; \zeta)=t \frac{d}{d \zeta} c(f(\zeta))+1 \geq-\frac{t}{T}+1>0
$$


and for all $(z, t) \in \Omega_{2}$, there is a unique solution $\zeta=\zeta(z, t)$ to (3.14). Notice that under the assumptions made on $c, f$, and $h,(3.14)$ have unique solutions $\tau$ and $\zeta$ in $\Omega_{1} \cup \Omega_{2}$, and that therefore $\tau=0 \Leftrightarrow \zeta=0$, since $c(h(0))=c(f(0))=z / t$.

Furthermore, it is clear that $u(0, t)=h(\tau(0, t))=h(t)$ for $t>0$, and $u(z, 0)=$ $f(\zeta(z, 0))=f(z)$ for $z>0$; so (3.15) satisfies the side conditions.

We are now ready to prove that the solution (3.15) also satisfies the partial differential equation. Differentiating (3.14) with respect to $z$, and then $t$, gives, respectively,

$$
\left\{\begin{array}{l}
\tau_{z}\left\{(t-\tau) c^{\prime}(h(\tau)) h^{\prime}(\tau)-c(h(\tau))\right\}=1 \\
\zeta_{z}\left\{t c^{\prime}(f(\zeta)) f^{\prime}(\zeta)+1\right\}=1
\end{array}\right.
$$

and

$$
\left\{\begin{array}{l}
\tau_{t}\left\{(t-\tau) c^{\prime}(h(\tau)) h^{\prime}(\tau)-c(h(\tau))\right\}=-c(h(\tau)) \\
\zeta_{t}\left\{t c^{\prime}(f(\zeta)) f^{\prime}(\zeta)+1\right\}=-c(f(\zeta))
\end{array}\right.
$$

Since, $(t-\tau) c^{\prime}(h(\tau)) h^{\prime}(\tau)-c(h(\tau)) \neq 0$ for $(z, t) \in \Omega_{1}$ and $t c^{\prime}(f(\zeta)) f^{\prime}(\zeta)+1 \neq 0$ for $(z, t) \in \Omega_{2}$, see (3.17) and (3.18), we get

$$
\left\{\begin{array}{l}
\tau_{z} c(h(\tau))+\tau_{t}=0 \\
\zeta_{z} c(f(\zeta))+\zeta_{t}=0
\end{array}\right.
$$

Therefore, finally from (3.15) it follows this solution satisfies

$$
\partial_{t} u+c(u) \partial_{z} u=\left\{\begin{array}{l}
\frac{\partial}{\partial t} h(\tau(z, t))+c(h(\tau(z, t))) \frac{\partial}{\partial z} h(\tau(z, t)) \\
\frac{\partial}{\partial t} f(\zeta(z, t))+c(f(\zeta(z, t))) \frac{\partial}{\partial z} f(\zeta(z, t))
\end{array}\right\}=0 \quad\left\{\begin{array}{l}
(z, t) \in \Omega_{1} \\
(z, t) \in \Omega_{2}
\end{array}\right.
$$

This theorem gives a maximal extension of the $(z, t)$-quarter plane for which no shocks in the solution $u^{+}$can occur. Sufficient conditions for no shocks to occur in $u^{+}$on the whole of the $(z, t)$-quarter plane was shown in Proposition 2.1 of (I). By noting the restriction on $c$, through Proposition 3.2, we observe that $\Omega_{1}$ is maximal if $h$ is increasing, and $\Omega_{2}$ is maximal if $f$ is decreasing.

In $\S 5$ the initial condition function, $f$, is assumed to be the zero function, and this means that the consistency condition may be violated as $h(0)$ may not be zero. This implies that a discontinuous jump in the solution from $u=0$, to $u=h(0)$, occurs on the initial causal wavefront, at time $z / c(h(0))$, so that the results of Theorem 3.1 hold for all time after this.

\section{Transmission problem}

The results of the previous section are applied to the pulse transmission problem, for specific cases of positively dissipative nonlinear optical media.

In a physical transmission problem an electromagnetic field is incident from the left onto an interface (the line $z=0$ ), of a half space, which is the Kerr medium $(z>0)$, see Figure 1. It is assumed that the initial conditions are $w_{1}(z, 0)=w_{2}(z, 0)=0$, inside the 
Kerr medium, $z>0$, for this problem. To the left of $z=0(z<0)$ only the $u^{+}$wave is present, and the results in $\S 3$ ensure that $u^{-}$is never excited for all $z$. Note in the physical variables

$$
\left(\begin{array}{l}
u^{+} \\
u^{-}
\end{array}\right)=\frac{1}{2}\left(\begin{array}{l}
g\left(w_{1}\right)-w_{2} \\
g\left(w_{1}\right)+w_{2}
\end{array}\right)
$$

so it is observed, that if $u^{-} \equiv 0$, then the two components $w_{1}$ and $w_{2}$ are related through

$$
w_{2}=-g\left(w_{1}\right)
$$

The $u^{+}$component can be physically measured from the fact that equation (4.1) implies $u^{+}=-w_{2}$. Remember that the $w_{2}$ component is related to the magnetic field intensity.

\subsection{Kerr polynomial medium}

As an explicit example, take the Kerr medium defined by the constitutive function

$$
F(E)=\epsilon_{1} E+\frac{1}{3} \epsilon_{3} E^{3} \Rightarrow F^{\prime}(E)=\epsilon_{1}+\epsilon_{3} E^{2}
$$

and therefore

$$
g^{\prime}(x)=\sqrt{\epsilon_{1}+\epsilon_{3} x^{2}}
$$

which, for positive constants $\epsilon_{1}$ and $\epsilon_{3}$, is bounded away from zero, and also with all higher derivatives bounded. Furthermore, integration gives

$$
g(x)=\frac{\epsilon_{1}}{2 \sqrt{\epsilon_{3}}} \ln \frac{x \sqrt{\epsilon_{3}}+\sqrt{\epsilon_{1}+\epsilon_{3} x^{2}}}{\sqrt{\epsilon_{1}}}+\frac{x \sqrt{\epsilon_{1}+\epsilon_{3} x^{2}}}{2}
$$

which is also bounded away from zero. Typical values for the parameters $\epsilon_{1}$ and $\epsilon_{3}$ are

$$
\left\{\begin{array}{l}
\epsilon_{1}=n_{0}^{2} \\
\epsilon_{3}=3 \mathcal{S} n_{0}^{4}
\end{array}\right.
$$

where the zero field refractive index is $n_{0}=1.5$, and the parameter $\mathcal{S}$ is in the range $10^{-14}$ $10^{-23} \mathrm{~m}^{2} / \mathrm{V}^{2}\left(\mathrm{SiO}_{2}: \mathcal{S}=1.3 \cdot 10^{-22} \mathrm{~m}^{2} / \mathrm{V}^{2}\right.$, and $\left.\mathrm{C}_{6} \mathrm{H}_{5} \mathrm{NO}_{2}: \epsilon_{3}=\sqrt{\epsilon_{1}} 2.89 \cdot 10^{-18} \mathrm{~m}^{2} / \mathrm{V}^{2}\right)$. The non-linear term is therefore usually very small, and propagation must occur over considerable distance for its effects on the propagating pulse to become very marked.

The Kerr model can have higher order dependence, on the field, as shown in the next equation for $g^{\prime}$. In that equation $g^{\prime}$ is shown with a term $\epsilon_{5}$, which allows for the possibility of fifth order dependence of $F$ on the electric field. This model is utilised in the numerical reconstruction results presented in $\S 5.1$.

$$
g^{\prime}(x)=\sqrt{\epsilon_{1}+\epsilon_{3} x^{2}+\epsilon_{5} x^{4}}
$$

It should be noted that unlike the third order model used earlier in this section this modified model will not have globally bounded derivatives.

It is convenient for numerical simulation to scale the problem through the scaling transformations: $\epsilon_{3} \rightarrow \epsilon_{3} / a^{2}, \epsilon_{5} \rightarrow \epsilon_{5} / a^{4}, t \rightarrow a t, z \rightarrow a z$, and $u^{+} \rightarrow a u^{+}$. This ensures for our simulations $z, t$ and the field amplitudes are very many orders of magnitude smaller than in a physical problem ${ }^{7}$.

Two different diagrammatic presentations of the propagating pulse, through this medium, are shown in Figures 2 and 3. Graphical representation of $g, g^{\prime}$ and the resultant wavespeed $c$, for this model, can be seen in Figures 7, 9 and 5, respectively.

\footnotetext{
${ }^{7}$ For the example of $\mathrm{SiO}_{2}$ with $\epsilon_{3}=0.1$, as used subsequently in this paper, then $a \approx 10^{-11}$.
} 


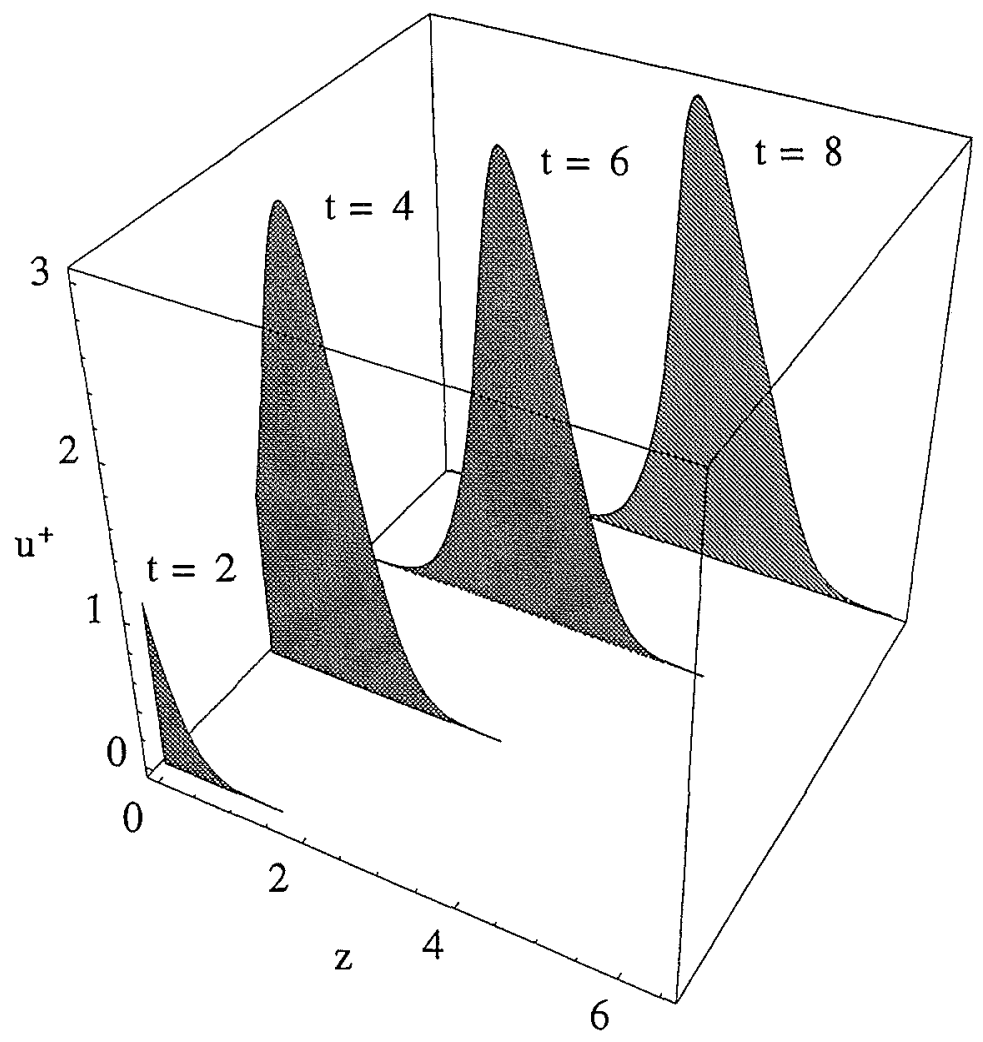

Figure 2: The propagation of an incident pulse $u^{+}(0, t)=3 H(t) \exp \left\{-(t-3)^{2}\right\}$ for the Kerr polynomial model with $\epsilon_{1}=1.5, \epsilon_{3}=0.1$ and $\epsilon_{5}=0$. Here, $H(t)$ denotes the Heaviside function and the initial condition is $f(z)=0$. Four different propagation times are shown, $t=2,4,6$, and 8 .

\subsection{Kerr saturation medium}

Often nonlinear effects saturate, and the model discussed in this section will allow this at field amplitudes set by the parameter $\beta$. The constitutive function is

$$
F(E)=\epsilon_{1} E+\frac{\alpha E^{3}}{E^{2}+\beta^{2}} \Rightarrow F^{\prime}(E)=\epsilon_{1}+\frac{\alpha E^{4}+3 \alpha \beta^{2} E^{2}}{\left(E^{2}+\beta^{2}\right)^{2}}
$$

and therefore

$$
g^{\prime}(x)=\sqrt{\epsilon_{1}+\frac{\alpha E^{4}+3 \alpha \beta^{2} E^{2}}{\left(E^{2}+\beta^{2}\right)^{2}}}
$$

Simailar scaling of the parameters, as carried out in the Kerr polynomial model, is performed on this model. It is seen for positive constants in the model, that $g^{\prime}$ is bounded away from zero and all higher order derivatives are also globally bounded.

A diagrammatic presentation of the propagating pulse through this medium, is shown in Figure 4. Graphical representation of $g, g^{\prime}$ and the resultant wavespeed $c$, for this model, can be seen in Figures 8, 10 and 6, respectively.

Observe, in Figures 2, 3, and 4 that the propagated pulses for $\ell=4.2$, and $\ell=0.6$, respectively, correspond to nearly the maximal existence distance for the classical solution 


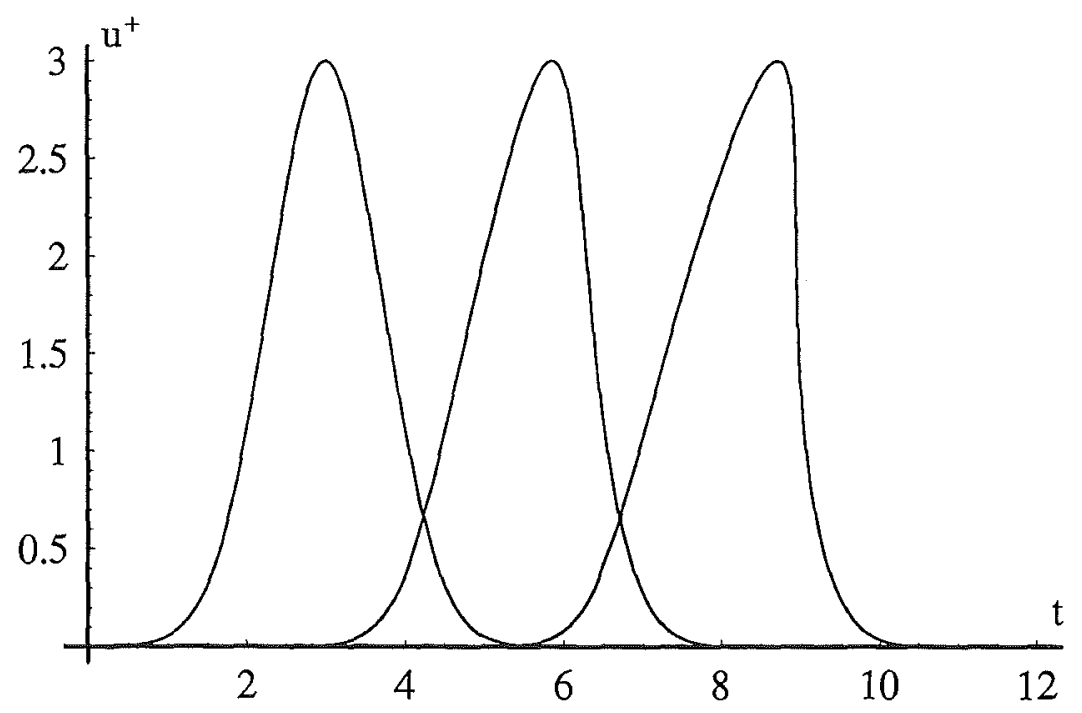

Figure 3: The propagation of an incident pulse $u^{+}(0, t)=3 H(t) \exp \left\{-(t-3)^{2}\right\}$ for the Kerr polynomial model with $\epsilon_{1}=1.5, \epsilon_{3}=0.1$ and $\epsilon_{5}=0$. The initial condition $f(z)=0$, and three different propagation distances, $z=\ell$ are shown, $\ell=0,2$, and 4.2.

of the one-way wave equation. For values of $z$ greater than this distance a shock forms, this can be seen to be almost occurring in these figures (the shock occurs in the Kerr polynomial and saturation model for $z$ equal to 4.23 and 0.65 , respectively). Also, observe that the near occurrence of a shock causes a steepening of the trailing edge of the pulse. One of the uses of Kerr media is to sharpen transmitted pulses. This steepening can be reasoned physically from the property of the wavespeed functional, as is proven in Proposition 3.2 and shown in 5, and 6 , the wavespeed increases with decreasing field amplitude. It follows that the characteristic traces steepen with decreasing field amplitude, and therefore as the traveling pulse decreases in amplitude, on the trailing edge of the pulse the lower amplitude values catch up with the higher amplitude parts! This should be contrasted with phenomena in which the wavespeed increases with increasing amplitude, so causing the shock to form on the leading edge. The reference (Remoissenet 1996) may be consulted to see that such leading edge shocks can occur in shallow-water waves ( $p 108$ ), and nonlinear transmission lines (p38).

\section{Inverse Problems}

Throughout this section it is assumed that $u^{+}(z, 0)=f(z)=0$, for $z>0$, and then the inverse transmission problem is posed as:

Given the initial boundary values as in equation (3.7) for a one-way wave equation (3.8), and the measured propagated field at $z=\ell$, namely $\bar{u}(t)=u^{+}(\ell, t)$, for $\ell / c(h(0))<t<T_{1}$, reconstruct the medium parameters. Specifically, in the first instance we reconstruct the wavespeed functional $c(\xi)$ for as large an interval of $\xi$ as possible. Here $T_{1}>\ell / c(h(0))$ is a time such that the interval $\left[\ell / c(h(0)), T_{1}\right]$ is non-zero. The time that the measurement of $\bar{u}$ starts, is after the initial wavefront arrives at $z=\ell$, namely $t=\ell / c(h(0))$.

It is required that the measured propagated solution is continuously differentiable for times after the initial wavefront has arrived, so that this imples $\ell<Z$ for all results in 


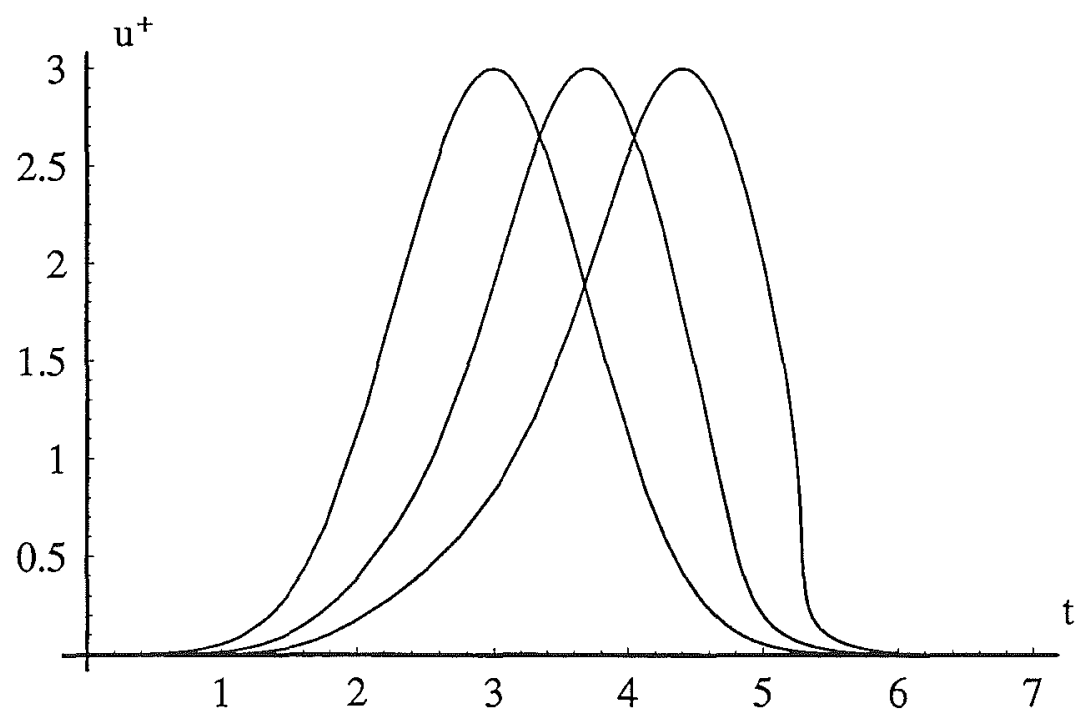

Figure 4: The propagation of an incident pulse $u^{+}(0, t)=3 H(t) \exp \left\{-(t-3)^{2}\right\}$. This is for the Kerr saturation model with $\epsilon_{1}=1, \alpha=4$ and $\beta=1$. Three different propagation distances, $z=\ell$, values are shown, with $\ell=0,0.3$, and $\ell=0.6$.

this section.

An implicit solution to equation (3.8) can then be written as

$$
u^{+}(z, t)=h\left(t-z / c\left(u^{+}\right)\right) \mathrm{H}\left(t-z / c\left(u^{+}\right)\right)
$$

rather than in the form of equation (3.13); here $\mathrm{H}$ denotes the Heaviside distribution. This equation is obtained by rearranging (3.11). Then an inverse reconstruction map is determined by the inverse of the map

$$
\bar{u}(t)=h(t-\ell / c(\bar{u})), \quad t>\ell / c(h(0))
$$

which is an implicit map from the wavespeed $c$ to the measurement $\bar{u}$. This equation can easily be inverted to read

$$
c(\bar{u})=\ell /\left(t-h^{-1}(\bar{u}(t))\right), \quad t>\ell / c(h(0))
$$

and this solution may also be written as

$$
c(\xi)=\ell /\left(\bar{u}^{-1}(\xi)-h^{-1}(\xi)\right), \quad t>\ell / c\left(\xi_{0}\right)
$$

with $t=\bar{u}^{-1}(\xi)$ and $\xi_{0}=h(0)$. To use this form of solution it is required that $\bar{u}$ is strictly monotone; this is in order to use its inverse function and that this can be the case is proven in (I). However, there, it is required that the function $h$ be monotone, which is not likely in an inverse experiment. The boundary function, $h$, is usually only locally monotone over an interval of $t \in \mathbb{R}$ It is shown here, that this implies that $\bar{u}$ is correspondingly locally monotone, and it follows that the existence of piecewise local inverses for $\bar{u}$ and $h$ is assured. The Lemma 2.2, from (I), is now generalised to prove that the transmission function $\bar{u}$ is piecewise locally monotone.

Lemma 5.1 The function $\bar{u}$ is piecewise strictly monotonic, with the assumptions that the functions $h$ and $c$ are continuously differentiable, also with $h$ piecewise strictly monotone, and $F$ is positively dissipative. 


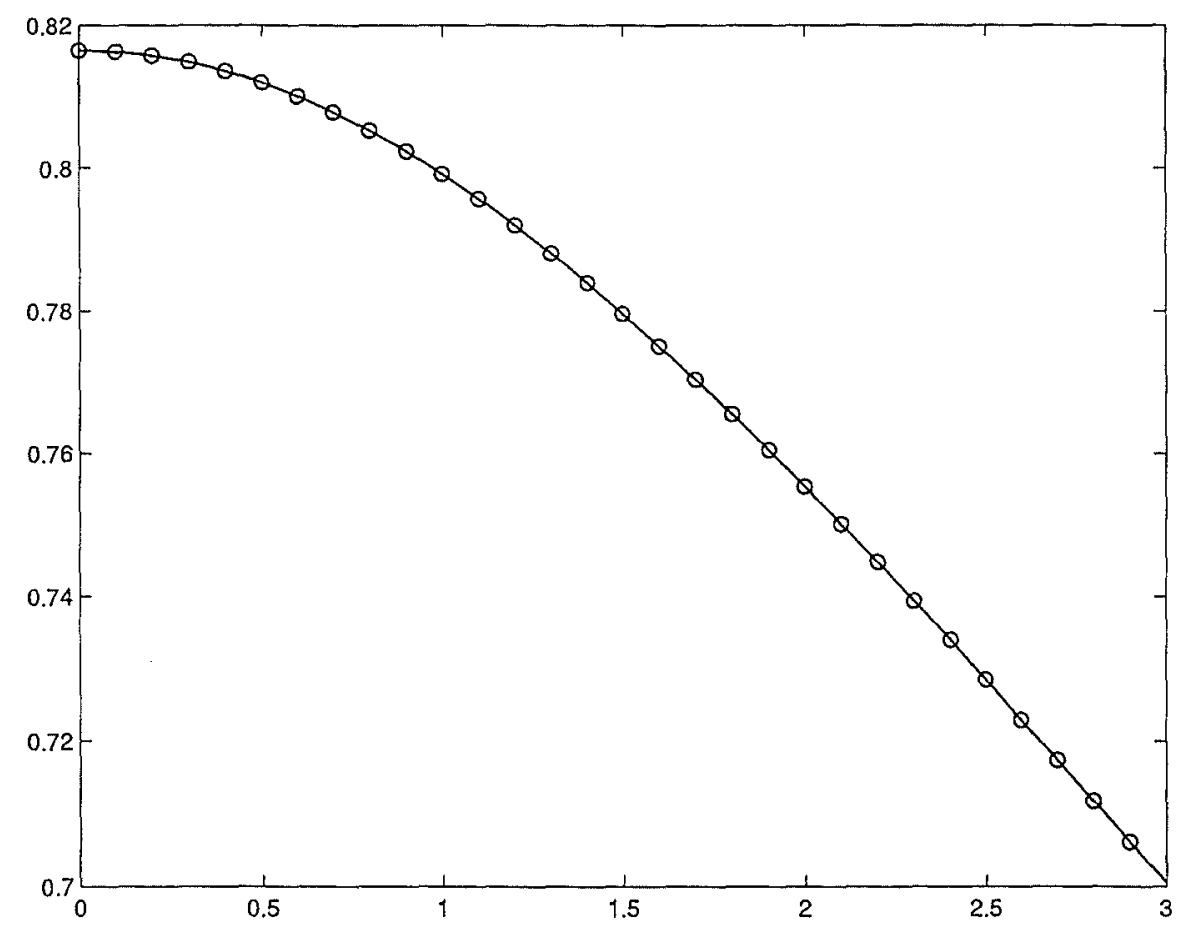

Figure 5: The true wavespeed functional $c(\xi) \longrightarrow$ and the reconstructed functional (circles) when the boundary value is $h(t)=3 H(t) \exp \left\{-(t-3)^{2}\right\}$. This is for the Kerr polynomial model with $\epsilon_{1}=1.5, \epsilon_{3}=0.1$ and $\epsilon_{5}=0$.

Proof: Upon differentiation of (5.1) with respect to $t$ it follows

$$
\bar{u}^{\prime}(t)=\frac{h^{\prime}(t-\ell / c(\bar{u}(t)))}{1-\ell c^{\prime}(\bar{u}(t)) h^{\prime}(t-\ell / c(\bar{u}(t))) / c^{2}(\bar{u}(t))}, \quad t>\ell / c\left(\xi_{0}\right)
$$

and if $h^{\prime}>0$, as $F$ is in the positively dissipative class by Proposition 3.2, $c^{\prime}<0$ and equation (5.4) implies $\bar{u}(t)$ is strictly increasing. Now if $h^{\prime}<0$ it follows $\bar{u}(t)$ is strictly decreasing, but with the possibility of a shock occurring; in particular when $h^{\prime}(t-\ell / c(\bar{u}))=$ $c^{2}(\bar{u}) /\left(\ell c^{\prime}(\bar{u})\right), c . f$. , equation (3.16), but because of our assumptions, this does not occur in $\Omega_{1}$.

The time values for which the measurement function $\bar{u}$ is strictly increasing, and decreasing, will not be the same as for $h$; see Figure 3. It should now be observed from Lemma 5.1 that if $h$ is strictly monotone on the interval $\left[0, T_{2}\right]$, then $\bar{u}$ will also be so on the interval $\left[\ell / c(h(0)), T_{2}+\ell / c\left(h\left(T_{2}\right)\right)\right]$; so implying that the locally monotone interval, for the two functions, will not be the same unless $c(h(0))=c\left(h\left(T_{2}\right)\right)$.

Corollary 5.1 The measurement function $\bar{u}$ is locally homeomorphic, when $h \in C^{1}$ and locally homeomorphic, provided $F$ is a positively dissipative function, and $c \in C^{1}$.

Proof: The results follows from Lemma 5.1 and elementary analysis.

It is seen that with the assumptions made, the solution of the inverse problem is unique and that the solution depends continuously upon the measured data, $\bar{u}$, provided this data is exact. 


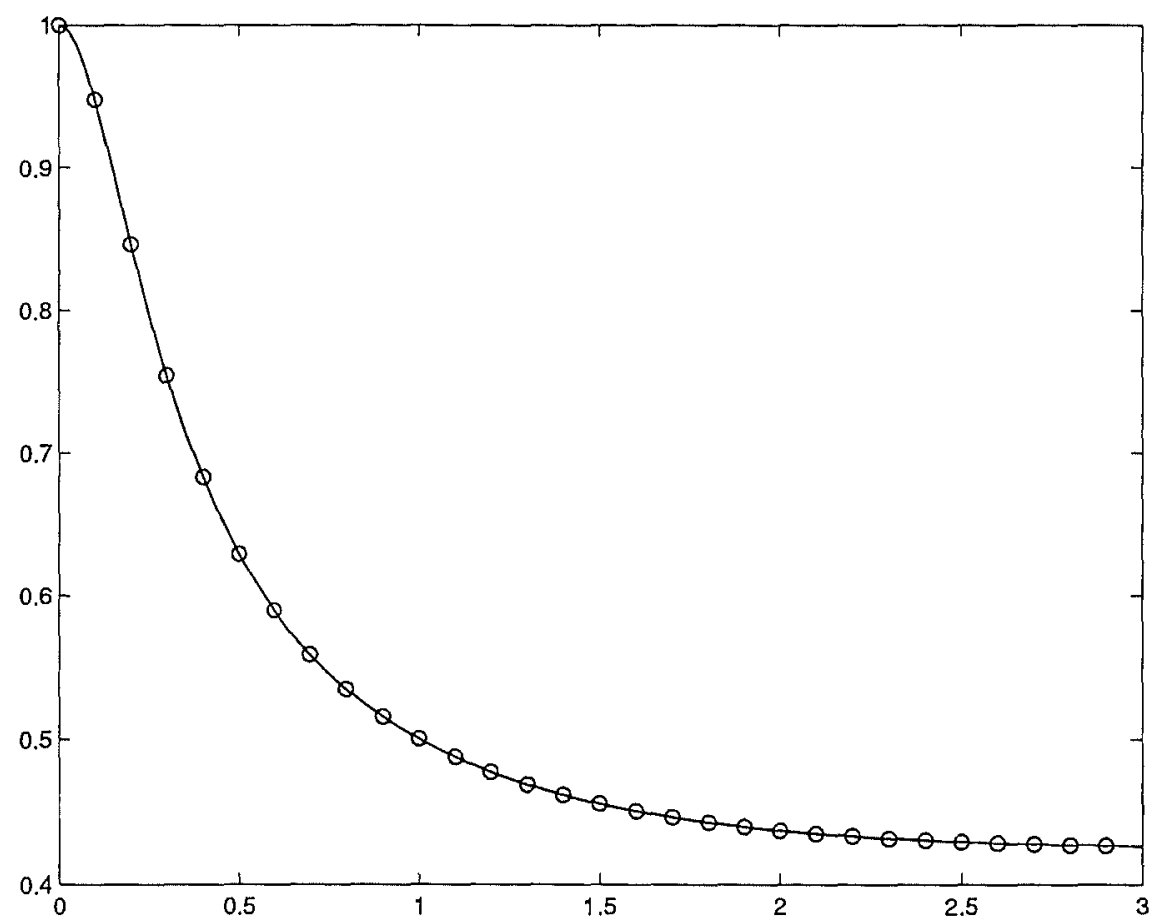

Figure 6: The true wavespeed functional $c(\xi)-$ and the reconstructed functional (circles) when the boundary value is $h(t)=3 H(t) \exp \left\{-(t-3)^{2}\right\}$. This is for the Kerr saturated model with $\epsilon_{1}=1.5, \alpha=4$, and $\beta=1$.

Theorem 5.1 The wave speed functional c $(u)$ can be uniquely reconstructed, from transmission data $\bar{u}$. Furthermore, the wavespeed functional depends continuously on the measurement $\bar{u}$ with a bounded Lipschitz continuity coefficient.

Proof: All that remains to be shown is the value of the Lipschitz constant. Differentiation of (5.2) with respect to $\bar{u}$ shows

$$
\frac{d}{d \bar{u}} c(\bar{u})=\frac{\ell}{\left(t-h^{-1}(\bar{u})\right)^{2}} \frac{d}{d \bar{u}} h^{-1}(\bar{u})
$$

so that the appropriate Lipschitz constant $\widetilde{C}$ is

$$
\widetilde{C}=\sup _{\xi}\left|\frac{\ell}{\left(t-h^{-1}(\xi)\right)^{2} h^{\prime}\left(h^{-1}(\xi)\right)}\right| \quad \text { with } t=\bar{u}^{-1}(\xi) \text {, and } \xi \in\left[\bar{u}(0), \bar{u}\left(T_{1}\right)\right]
$$

It follows that the Lipschitz constant exists, and is bounded for a positively dissipative medium provided $h^{\prime} \neq 0$ with $(\ell, t) \in \Omega_{1}$.

It is shown in $\S 3$ that the function $g$ can be related to the wave speed though the initial value differential equation, see (3.5)

$$
\left\{\begin{array}{l}
\frac{d}{d \xi} g^{-1}(\xi)=c(\xi) / c_{0} \\
g^{-1}(0)=0
\end{array}\right.
$$


It follows that once the wavespeed has been reconstructed, $g^{-1}$ can be found uniquely, so enabling $g$ to be calculated over the region for which $g^{-1}$ is injective ${ }^{8}$, and for which $c$ is known.

Corollary 5.2 The material function $g(\xi)$ can be uniquely reconstructed from the transmission data, and it depends continuously on the measurement data $\bar{u}$, this is provided this data is exact.

\subsection{Numerical Results}

All the inverse reconstruction experiments were carried out with an incident pulse

$$
h(t)=3 H(t) \exp \left\{-(t-3)^{2}\right\}
$$

and this function is piecewise monotone, in two pieces, and satisfies the conditions required in the previous section. Note that $h(0) \neq 0$ for this incident wave and therefore a finite jump discontinuity propagates along the characteristic determined by $c(h(0))=z / t$.

The solution to the direct transmission problem, as given by the implicit solution (5.1), was found, as displayed in Figures 2-4, by first solving the nonlinear equation (3.11), and then substituting into (3.13). This yields a unique solution up to the point $z=Z$, as proven in Theorem 3.1. The transmitted fields $\bar{u}$ for the two models considered in this paper, namely a Kerr polynomial medium, and a Kerr saturated medium, are shown in Figures 3-4 for various propagation lengths.

We recap the assumptions made for the inverse reconstruction problem:

- Measure the transmitted field where this field is assumed to be continuous, i.e., no shock has occurred.

- The incident pulse, $h(t)$, is known, piecewise strictly monotone, and it is continuously differentiable for $t \in\left[0, T_{1}\right]$.

- The transmission length $\ell$ is known. If this is not the case it will only effect the scaling of $c$ and subsequent parameters. The functional form of $c$ can still be found.

- The free space wavespeed $c_{0}$ is known.

For the inverse reconstruction problem $h$ is known, so that the inverse of this function $h^{-1}$ can be found over an appropriate interval $1^{9}$. Lemma 5.1 ensures that the inverse function to the measured transmission function, namely $\bar{u}^{-1}$ can be found over an appropriate interval. Once these inverse functions have been calculated the equation (5.3) provides the solution. The given boundary function $h$ has two monotone sections, $0<t<3$, and $3<t<6$, so that (5.3) can be used over two separate intervals. When these two intervals are used for the non-noisy data, as used here, this yields identical results for $c$. However, for noisy data by performing two reconstructions this would enable some smoothing to be achieved. Figures 5 and 6 show the reconstructed wavespeed when $\ell=4.2$, and $\ell=0.6$, for the Kerr polynomial, and the Kerr saturated models, respectively. The results when using transmission data at $\ell=2$, and $\ell=0.3$, for the Kerr polynomial, and the Kerr saturated model, respectively, cannot be distinguished from these. Note in these figures

\footnotetext{
${ }^{8}$ Which by Proposition 3.1 is $\mathbb{R}$.

${ }^{9}$ The inverse function $h^{-1}$ is found numerically by solving the nonlinear equation $h(\xi)-y=0$, for $\xi$ given $y$, with the inverse function defined through $\xi=h^{-1}(y)$. A similar method is used for $\bar{u}^{-1}$.
} 


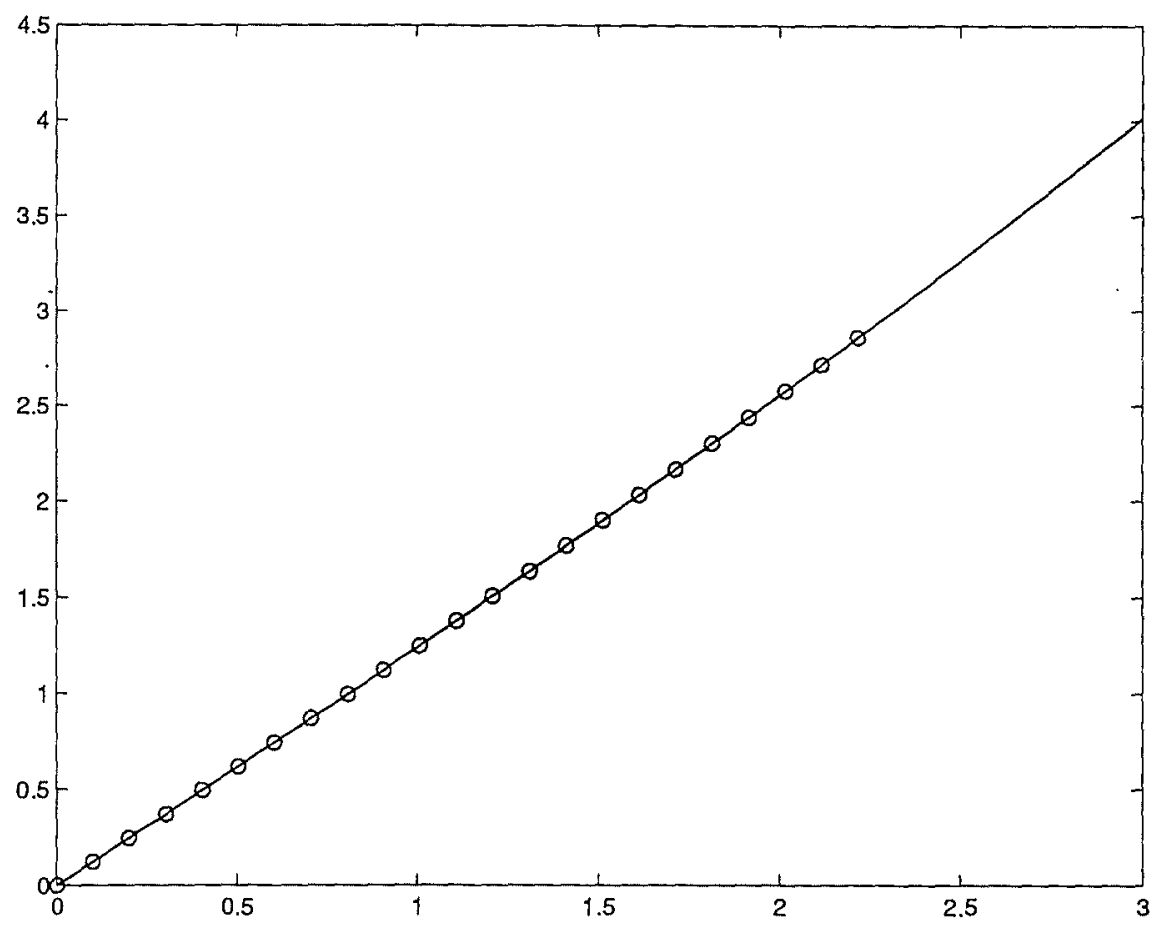

Figure 7: The true $g(\xi)$ function —- and the reconstructed function (circles) when the boundary value is $h(t)=3 H(t) \exp \left\{-(t-3)^{2}\right\}$. This is for the Kerr polynomial model with $\epsilon_{1}=1.5, \epsilon_{3}=0.1$ and $\epsilon_{5}=0$.

that $c(\xi)$ can only be found for $0<\xi<3$ as the applied boundary function only has this range.

In Figures 7, and 8, the reconstructed $g$ function is shown for the Kerr polynomial $(\ell=4.2)$ model, and the Kerr saturated model $(\ell=0.6)$, respectively. Identical results are obtained when $\ell=0.3$ and $\ell=2$ for these respective models. Note in these figures that $g(\xi)$ can only be found for $0<\xi<\xi_{a}$, where $\xi_{a}=g^{-1}(3)$, as the applied boundary function only has this range.

To reconstruct the material parameters $\left\{\epsilon_{1}, \epsilon_{3}, \epsilon_{5}\right\}$, and $\left\{\epsilon_{1}, \alpha, \beta\right\}$, for the respective models, it is more convenient to work with $g^{\prime}(\xi)$ as given by equations (4.3), and (4.4), respectively, to find the coefficients. Figures 9 and 10 show the true and reconstructed Kerr polynomial and saturated Kerr model $g^{\prime}$ functions, respectively.

Let $\Sigma$ denote the set of these coefficients, and $\widetilde{g}^{\prime}$ denote the reconstructed $g^{\prime}$ function. As $g^{\prime}$ denotes the model representation given by equations (4.3) and (4.4), the appropriate coefficients can be calculated by the maximum likelihood principle. If $\widetilde{g}^{\prime}$ has been reconstructed at $\xi_{i}, i=0 \ldots N$, values of $\xi$ through the previously specified algorithm, and if $\|\cdot\|_{2}$ denotes the standard discrete $\ell_{2}$-norm over the $N+1$ values of $\xi_{i}$, then the problem of finding the maximum likelihood probability for the coefficients can be written as

$$
\min _{\Sigma}\left\|\widetilde{g}^{\prime}-g^{\prime}\right\|_{2}
$$

Where in this equation $g^{\prime}$ is the analytical representation of the model; either (4.3) or (4.4). For the model given by equation (4.3) the method of the least sum of the squares 


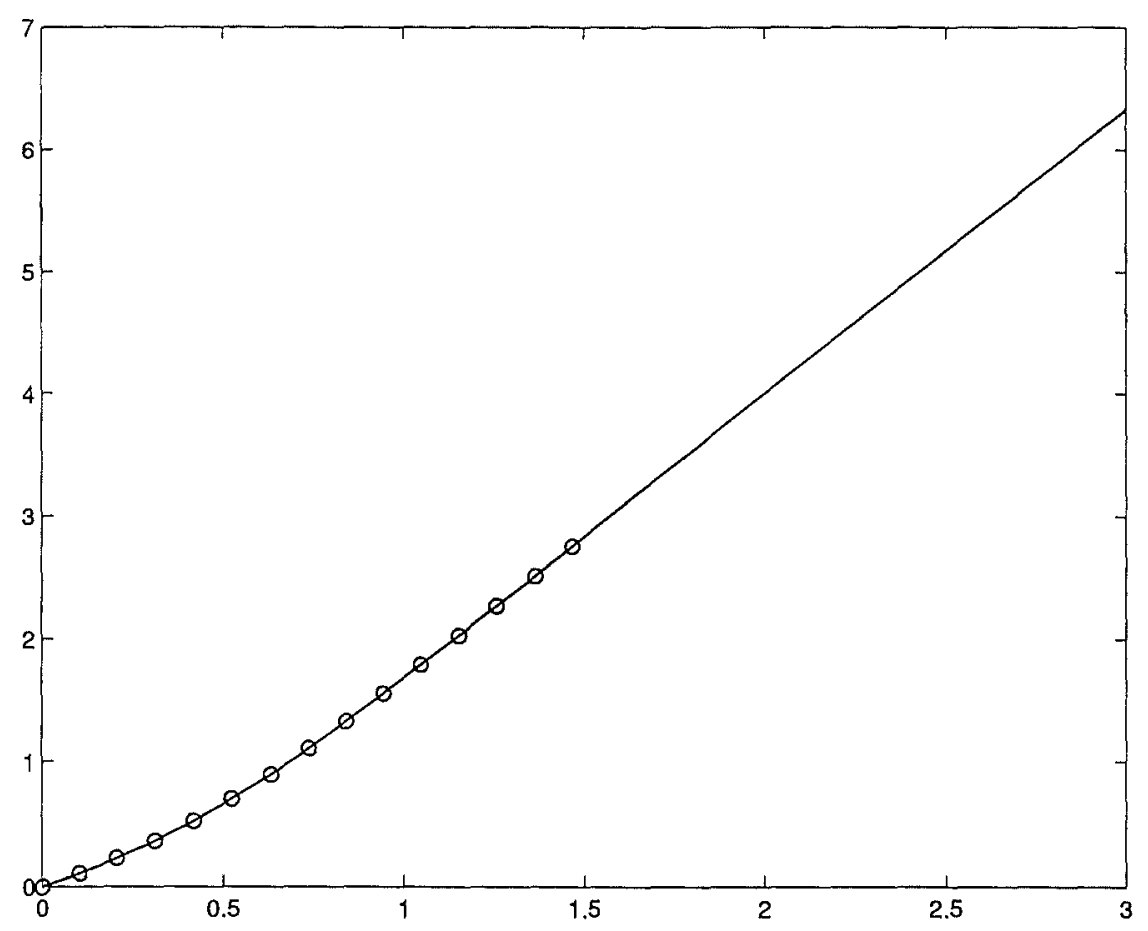

Figure 8: The true $g(\xi)$ function — and the reconstructed function (circles) when the boundary value is $h(t)=3 H(t) \exp \left\{-(t-3)^{2}\right\}$. This is for the Kerr saturated model with $\epsilon_{1}=1.5, \alpha=4$, and $\beta=1$.

of the errors problem is linear ${ }^{10}$, and it is a standard regression problem. However, for equation (4.4) the method of least squares problem is a nonlinear regression problem. For the reconstructed $\widetilde{g}^{\prime}$ functions shown in Figures 9 and 10, the respective parameters, were found to be

Fig $9 \epsilon_{1}=1.5, \epsilon_{3}=1.001, \epsilon_{5}=0$ with an $\ell_{2}$-error of $3.1 \times 10^{-4}$.

Fig $10 \epsilon_{1}=1.0004, \alpha=4.0006, \beta=1.0015$ with an $\ell_{2}$-error of $1.1 \times 10^{-3}$.

If the the wrong choice of models is made, and an attempt is made to fit a Kerr polynomial model to the saturated $\widetilde{g}^{\prime}$ data, values of a polynomial were found that were not positively dissipative, and a $\ell_{2}$-error of 1.2 was obtained; so illustrating a false premise. Similarly, if an attempt is made to fit the saturated model to the reconstructed $\widetilde{g}^{\prime}$ data from the Kerr polynomial problem, then a least squares error measure of $9.1 \times 10^{-4}$ was found; this was not a clear false premise. This can be attributed to a well known result in approximation theory; the fact that a rational approximation can provide a wider class of function fit than a polynomial.

\section{Conclusions}

This paper has shown that it is possible to reconstruct the material parameters for a nonlinear medium, from measurement of the propagated electromagnetic pulse through

\footnotetext{
${ }^{10}$ If the fit is made to $g^{\prime 2}$.
} 


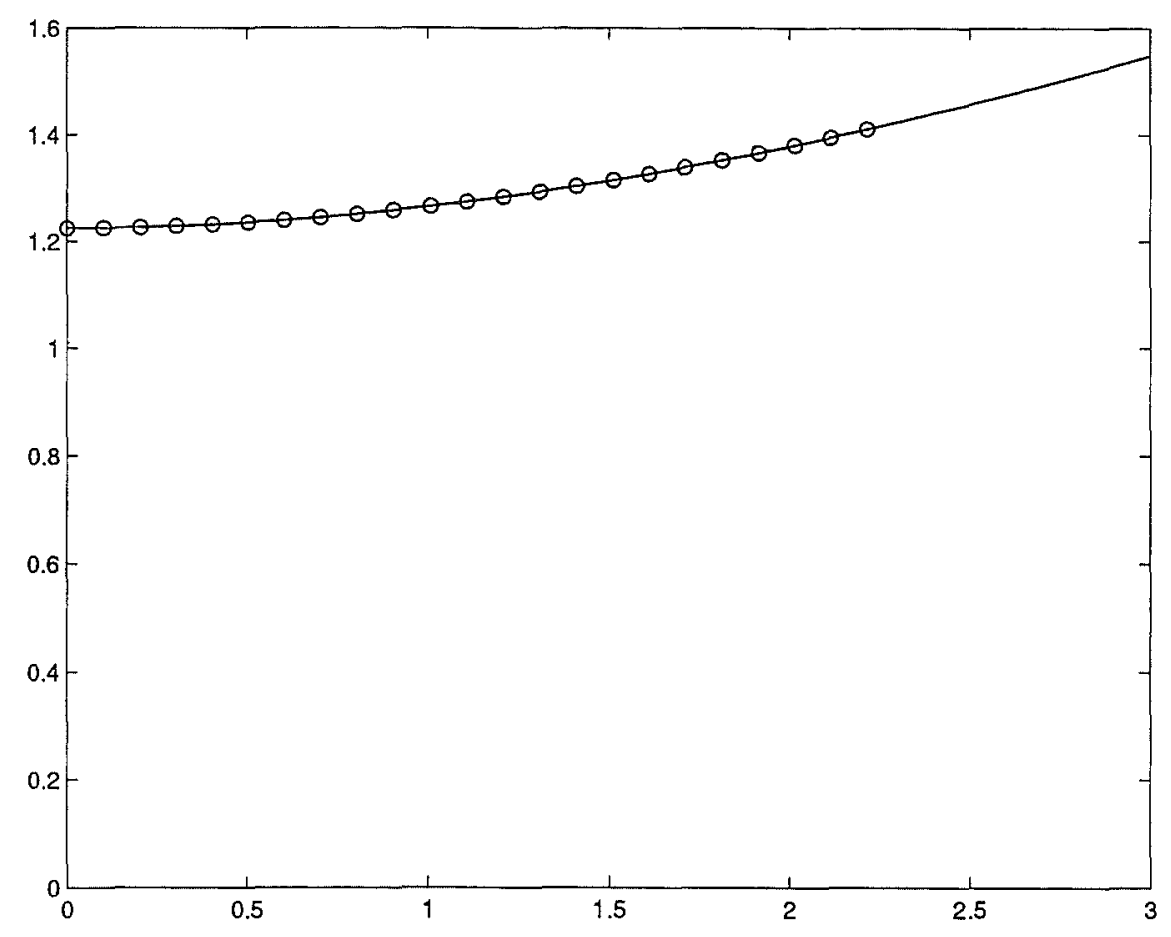

Figure 9: The true $g^{\prime}(\xi)$ function — and the reconstructed function (circles) when the boundary value is $h(t)=3 H(t) \exp \left\{-(t-3)^{2}\right\}$. This is for the Kerr polynomial model with $\epsilon_{1}=1.5, \epsilon_{3}=0.1$ and $\epsilon_{5}=0$.

the medium. The inverse algorithm is not model dependent and therefore useful if the underlying nonlinear model of the material is not known. Specifically, we have illustrated the use of the algorithm for the Kerr medium. Furthermore, the solution depends continuously on the measurement data. This is a rare occurrence in an inverse problem. However, it should be noticed, that in optics the field quantities are not directly accessible, but only observable through a functional.

\section{Acknowledgment}

One of the authors (G.K.) wishes to express his gratitude to the Department of Mathematics, University of Canterbury, Christchurch, New Zealand for the Visiting Erskine Fellowship that initiated this project and collaboration. The work reported in this paper is partially supported by a grant from the Swedish Research Council for Engineering Sciences and their support is gratefully acknowledged.

\section{Appendix A Uniqueness result}

In this appendix it is proven, following (Kreiss and Lorenz 1989, Chapter 5), that the direct problem posed in $\S 3$ has at most one classical solution. The requirement of global boundedness of $c$ needed for this result is true for the Kerr and saturated models used in this paper. 


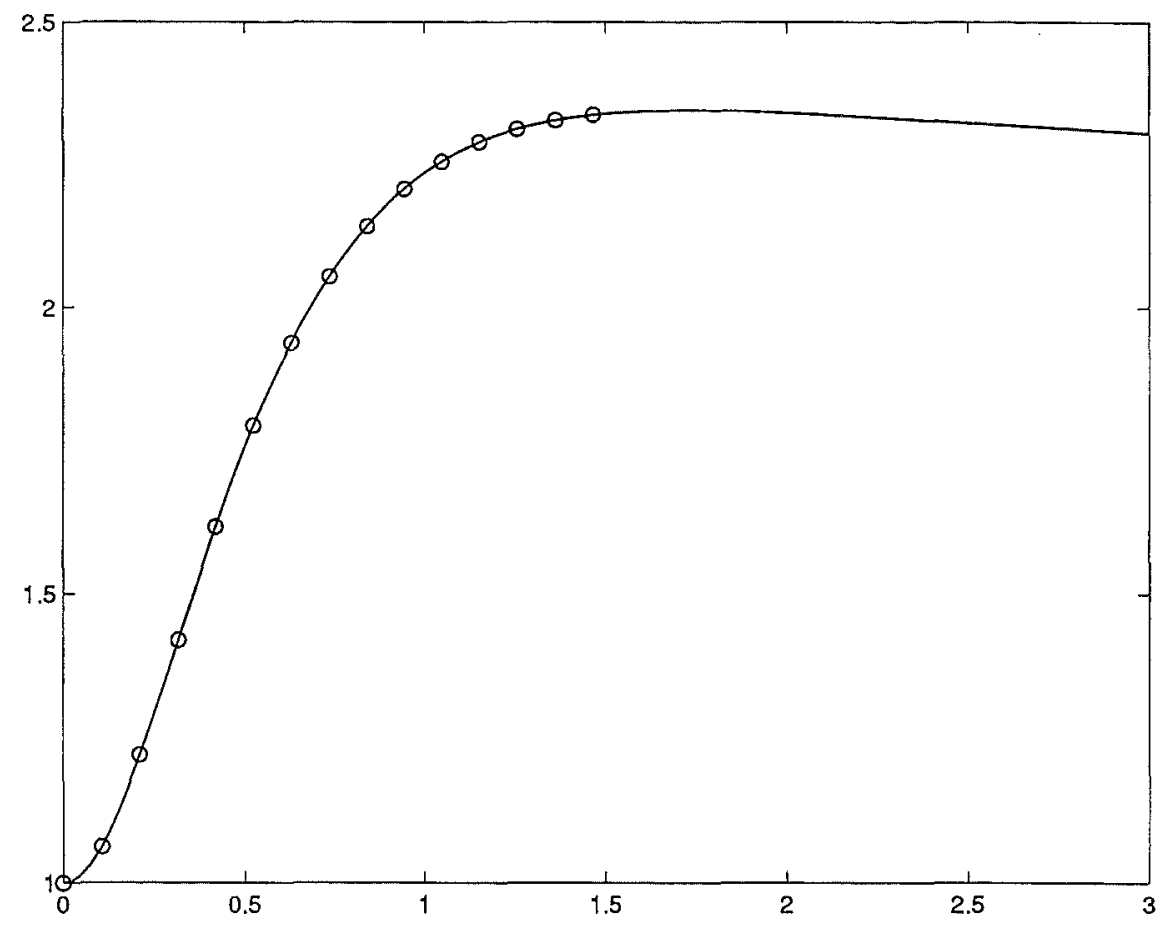

Figure 10: The true $g^{\prime}(\xi)$ function - and the reconstructed function (circles) when the boundary value is $h(t)=3 H(t) \exp \left\{-(t-3)^{2}\right\}$. This is for the Kerr saturated model with $\epsilon_{1}=1.5, \alpha=4$, and $\beta=1$.

Theorem A.1 Let the matrix $A(u) \in \mathbb{R}^{2,2}$ be diagonal of the form

$$
A(u)=c(u)\left(\begin{array}{cc}
-\lambda_{1} & 0 \\
0 & \lambda_{2}
\end{array}\right)
$$

where $\lambda_{1}>0, i=1,2$, and let $c$ be bounded and have bounded first order derivatives, i.e., $\|A\|_{\infty}<\infty,\|D A\|_{\infty}<\infty$. Then the initial-boundary value problem for $u=\left\{u^{+}, u^{-}\right\}$ satisfying

$$
\partial_{t}\left(\begin{array}{l}
u^{+} \\
u^{-}
\end{array}\right)=A(u) \partial_{z}\left(\begin{array}{l}
u^{+} \\
u^{-}
\end{array}\right), \quad\left\{\begin{array}{l}
t>0 \\
z>0
\end{array}\right.
$$

subject to

$$
\left\{\begin{array}{l}
u^{ \pm}(z, 0)=f^{ \pm}(z), \quad z \geq 0 \\
u^{+}(0, t)=h(t), \quad t \geq 0 \\
\lim _{z \rightarrow \infty} u^{ \pm}(z, t)=0 \text { for all } t \geq 0
\end{array}\right.
$$

has at most one classical solution.

Proof: From the assumptions we have that

$$
\|A(u)-A(v)\| \leq C_{1}\|u-v\|
$$


Now suppose $u$ and $v$ are two solutions and denote $\boldsymbol{w}=\left\{w^{+}, w^{-}\right\}=u-v$. Furthermore, $\boldsymbol{w}$ satisfies the side conditions

$$
\left\{\begin{array}{l}
w^{ \pm}(z, 0)=0, \quad z \geq 0 \\
w^{+}(0, t)=0, \quad t \geq 0 \\
\lim _{z \rightarrow \infty} w^{ \pm}(z, t)=0 \text { for all } t \geq 0
\end{array}\right.
$$

Then

$$
\partial_{t} w=A(u) \partial_{z} u-A(v) \partial_{z} v=A(u) \partial_{z} w+(A(u)-A(v)) \partial_{z} v
$$

and it follows that ${ }^{11}$

$$
\frac{1}{2} \frac{d}{d t}(w, w)=\left(w, w_{t}\right)=\left(w, A(u) w_{z}\right)+\left(w,(A(u)-A(v)) v_{z}\right)
$$

Integration by parts gives, due to the homogeneous boundary condition of $w^{+}$

$$
\left(w, A(u) w_{z}\right)=-c(u(z=0)) \lambda_{2}\left\|w^{-}(z=0)\right\|^{2}-\left(w_{z}, A(u) w\right)-\left(w, A(u)_{z} w\right)
$$

which implies

$$
\begin{aligned}
\left(\boldsymbol{w}, A(u) w_{z}\right) & =-\frac{1}{2}\left(\boldsymbol{w}, A(\boldsymbol{u})_{z} \boldsymbol{w}\right)-\frac{1}{2} c(\boldsymbol{u}(z=0)) \lambda_{2}\left\|w^{-}(z=0)\right\|^{2} \\
& \leq-\frac{1}{2}\left(\boldsymbol{w}, A(\boldsymbol{u})_{z} \boldsymbol{w}\right) \leq C_{2}\|\boldsymbol{w}\|^{2}
\end{aligned}
$$

Therefore we get

$$
\frac{d}{d t}\|w\|^{2} \leq C_{3}\|w\|^{2}
$$

so by Grönwall's lemma, and the initial conditions, this inequality proves that $\|w\|=0$, for all $t \geq 0$, and the theorem is proven.

\section{References}

Åberg, I., G. Kristensson, and D. J. N. Wall (1996). Transient waves in non-stationary media. J. Math. Phys. 37(5), 2229-2252.

Agrawal, G. P. (1995). Nonlinear Fibre Optics. New York: Academic Press.

Connolly, T. J. and D. J. N. Wall (1997). On some inverse problems for a nonlinear transport equation. Inverse Problems 13(2), 283-295.

Karlsson, A. and G. Kristensson (1989). Constitutive relations, dissipation and reciprocity for the Maxwell equations in the time domain. Technical Report LUTEDX/(TEAT-7005)/1-35/(1989), Lund Institute of Technology, Department of Electromagnetic Theory, P.O. Box 118, S-211 00 Lund, Sweden.

${ }^{11}$ The scalar product is in the spatial variable $z$, i.e.,

$$
(u, v)=\int_{0}^{\infty}\left(u_{1}(z) v_{1}(z)+u_{2}(z) v_{2}(z)\right) d z, \quad\|u\|=\sqrt{(u, u)}
$$


Kreiss, H.-O. and J. Lorenz (1989). Initial-Boundary Value Problems and the NavierStokes Equations. San Diego: Academic Press.

Landau, L., E. Lifshitz, and L. Pitaevskĭ (1984). Electrodynamics of Continuous Media. Oxford: Pergamon.

Remoissenet, M. (1996). Waves called Solitons - Concepts and Experiments (2 ed.). Berlin: Springer-Verlag.

Shen, Y. R. (1995). Principles of Nonlinear Optics. New York, N.Y.: John Wiley \& Sons, Inc.

Shubert, M. and B. Wilhelmi (1986). Nonlinear Optics and Quantuum Electronics. New York, N.Y.: John Wiley \& Sons, Inc.

Wall, D. J. and J. Lundstedt (1996). Inverse source problems involving the one-way wave equation: source function reconstruction. Technical Report $146 / 1-26 /(1996)$, Canterbury University, Christchurch 1, New Zealand. 